\title{
Relacionando Conceitos de Áreas de Estudo de Desempenho da Ciência da Computação
}

\author{
Rafael de Souza Stabile, Sarita Mazzini Bruschi, Paulo Sérgio Lopes de Souza \\ ${ }^{1}$ Departamento de Sistemas de Computação - Instituto de Ciências Matemáticas \\ e de Computação - Universidade de São Paulo (USP) - São Carlos - SP - Brasil \\ stabilerafael@usp.br, \{sarita, pssouza\}@icmc.usp.br
}

\begin{abstract}
There are several areas of computer science whose main point of study is the performance of computer systems, whether for the construction of software with performance as to estimate the performance of systems. Examples of such areas are: Performance Evaluation, Software Performance Engineering, Software Performance Testing and Experimental Studies. However, there is no work in the literature that unifies or even lists the concepts and methodologies they raised. In this context, this paper describes how these areas can be related, and this study will provide the basis for the definition of an ontology.
\end{abstract}

Resumo. Existem diversas áreas da Ciência da Computação que têm como ponto principal o estudo de desempenho de sistemas computacionais, seja para a construção de softwares com desempenho, como para estimar o desempenho de sistemas. Exemplos dessas áreas são: Avaliação de Desempenho, Engenharia de Desempenho de Software, Teste de Desempenho de Software e Estudos Experimentais. Porém, não existe na literatura um trabalho que unifique ou mesmo relacione os conceitos e metodologias por elas abordados. Neste contexto, este artigo descreve como essas áreas podem ser relacionadas, estudo este que servirá de base para a definição de uma ontologia.

\section{Introdução}

Dentro da grande área da Ciência da Computação, sistemas corretos e eficientes são pesquisados exaustivamente, com critérios e metodologias próprias de diferentes áreas. Algumas dessas áreas são: Teste de Software, que tem como objetivo encontrar defeitos no software; Teste de Desempenho de Software, que tem como objetivo encontrar erros relacionados ao desempenho do software; Avaliação de Desempenho, que busca quantificar o desempenho de sistemas computacionais; Engenharia de Desempenho de Software, que tem como objetivo fornecer métodos para construir softwares com desempenho; e Estudos Experimentais, que proporciona metodologias para execução de experimentos.

Considerando a existência dessas diversas áreas, percebe-se que elas não apresentam um vocabulário unificado a respeito de seus termos e métodos, resultando em uma replicação de esforços de especialistas dessas diferentes áreas, que na maioria das vezes têm o mesmo objetivo.

Neste contexto, o objetivo deste artigo é descrever como as áreas citadas no início dessa seção podem ser relacionadas, evidenciando essa relação. Para a realização do trabalho apresentado neste artigo, as áreas foram estudas e a partir desse estudo foram 
construídos e validados mapas conceituais para cada delas. Todos os termos que apareceram nos mapas conceituais foram listados e aqueles que se repetiam em mais de uma área foram destacados.

O relacionamento apresentado neste artigo está inserido em um projeto maior, que tem como objetivo unificar os conceitos de desempenho presentes na literatura de cada uma das áreas apresentadas anteriormente através de uma ontologia, proporcionando uma maneira unificada de se analisar o desempenho de sistemas computacionais.

Na Seção 2 são descritos os trabalhos relacionados e na Seção 3 as áreas estudadas. Na Seção 4 é apresentado o relacionamento proposto para essas áreas e na Seção 5 são apresentadas as próximas etapas a serem seguidas neste trabalho. Por fim, a Seção 6 apresenta as conclusões.

\section{Trabalhos Relacionados}

Existem diversos trabalhos que têm como objetivo reunir o conhecimento isolado das áreas já apresentadas, utilizando para isso ontologias.

Em [Sancho et al. 2007] é proposto um conjunto de regras semânticas a fim de formalizar o conhecimento sobre a Engenharia de Desempenho de Software, propondo uma base de dados ontológica que contém todo o conteúdo dessa área. Já [Freitas and Vieira 2014] apresenta uma ontologia para representar o conhecimento sobre Teste de Desempenho, guiando assim o testador sobre como proceder para a realização de tais testes. Já [Cortellessa 2005] discute se é possível a existência de uma ontologia que contenha os conceitos e relações a respeito de desempenho de software.

\section{3. Áreas de Estudo}

\subsection{Avaliação de Desempenho}

A avaliação de desempenho de sistemas computacionais é crucial, por exemplo, na escolha de qual o melhor ambiente computacional para um determinado cenário. Para se realizar uma Avaliação de Desempenho, é necessário seguir três etapas, sendo elas: Planejamento de Experimentos, escolha da Técnica de Avaliação, e Análise dos Resultados.

O objetivo do planejamento de experimentos é obter o máximo de informação com um número mínimo de experimentos, e com os resultados conseguir separar os efeitos de vários fatores no resultado observado, e determinar o quão significante é o efeito dos fatores nos resultados observados [Jain 1991]. Já as técnicas de avaliação se diferem em como são coletadas as informações referentes aos parâmetros requeridos para a avaliação. As técnicas podem ser divididas em dois grupos, sendo: baseadas na Aferição (informações são colhidas do próprio sistema), ou baseadas na Modelagem (informações através de modelos que representam o sistema os quais podem ser resolvidos através de simulações, ou ainda analiticamente). [Jain 1991].

\subsection{Engenharia de Desempenho de Software}

A Engenharia de Desempenho do Software (EDS) é uma coletânea de métodos para desenvolver softwares que tem como objetivo oferecer a resposta de execução do software com o desempenho requerido pelo usuário [Smith 1986], analisando o desempenho do 
software durante seu ciclo de vida. Seus métodos envolvem desde a utilização de processos de criação provindos da Engenharia de Software, até análises de desempenho durante a criação do software, como construção e análises de modelos, utilizando, por exemplo, simulação.

Os métodos da Engenharia de Desempenho de Software podem ser divididos nos que são baseados na medição e nos que são baseados em modelos [Woodside et al. 2007]. Especificamente para a EDS aqueles baseados em medição não são muito utilizados, já que é necessário que o software esteja pronto. Segundo [Smith 1986] tais técnicas são utilizadas na fase de teste de integração do sistema, e resulta em maiores custos para o desenvolvimento e manutenção. As metodologias baseadas em modelos têm como objetivo construir primeiramente um modelo do sistema, testar seu desempenho, e então realizar a implementação.

\subsection{Teste de Desempenho de Software}

O Teste de Desempenho de Software é um tipo de Teste de Software que tenta encontrar possíveis defeitos de desempenho do programa. Tais defeitos podem ser gargalos, ou mesmo um conjunto de linhas de código que levem a degradação do desempenho da execução do software.

O objetivo é determinar ou validar a Capacidade de resposta, a Vazão, a Confiabilidade e a Escalabilidade de um sistema quando submetido a uma determinada carga [Freitas and Vieira 2014]. O teste de desempenho define características de desempenho do software afim de encontrar problemas relacionados ao seu desempenho e então permitir que tal sistema seja otimizado.

\subsection{Estudos Experimentais}

Estudos Experimentais é uma subárea da Engenharia de Software que tem como objetivo caracterizar, avaliar, prever ou mesmo melhorar produtos, processos, recursos ou modelos. Nela são desenvolvidos uma série de métodos que guiam um experimento que necessite ser realizado. Como é possível perceber, esta não é uma área que está relacionada diretamente com o desempenho. Porém tem correlação com o planejamento e condução de experimentos, algo imprescindível para a análise de um sistema computacional.

A metodologia para a execução de experimentos compreende cinco passos: definição do experimento expresso em termos de problemas e objetivos; planejamento, ou seja, determinação do projeto levando em conta a validade do experimento; execução e coleta de dados; análise e interpretação dos resultados; apresentação e empacotamento dos resultados [Wohlin et al. 2012].

\section{Relacionamento entre áreas}

Não existe na literatura um trabalho que unifique os conceitos das áreas apresentadas neste artigo, ou mesmo que apresente uma relação entre eles. Para apontar que existe uma correlação entre tais áreas foram feitos estudos de cada uma delas. É importante salientar que, para os estudos feitos, foram seguidas indicações de leitura por especialistas das áreas envolvidas neste projeto. A fim de melhor identificar conceitos de cada área e relações entre elas, foram desenvolvidos mapas conceituais de cada uma delas ${ }^{1}$.

\footnotetext{
${ }^{1}$ Por questões de limitação de espaço no artigo, os mapas conceituais foram omitidos do artigo. Para ter acesso a esses mapas entre em contato com os autores.
} 
Para cada mapa conceitual feito, o mesmo passou por validação de especialistas nas áreas envolvidas que o mesmo aborda. Tais especialistas são professores do Instituto de Ciências Matemáticas e Computação (ICMC-USP), alunos do programa de pósgraduação em Ciências de Computação e Matemática Computacional do ICMC-USP, ou colaboradores que tenham experiência nas áreas envolvidas.

A partir dos mapas foi possível apontar conceitos que são equivalentes (mesmo nome) entre as áreas de estudo deste projeto, ou que tenham pelo menos alguma relação em seu significado.

A partir da identificação de conceitos, a relação entre eles e suas equivalências, caso existam, foi possível relacionar as áreas de estudo que envolvem o desempenho, apontando, dessa forma, os métodos e termos pertencentes em cada uma dessas áreas, e aqueles que pertencem a mais de uma área. Dessa forma, foi feita uma lista com todos os termos que fazem parte de todos os mapas, e aqueles que se repetiam em mais de um mapa também foram apontados.

Observando as áreas como conjuntos de termos e métodos, foi possível perceber que existem intersecções, sendo algumas específicas entre duas áreas.

É possível observar que a área de Avaliação de Desempenho tem intersecção com as áreas de Teste de Desempenho de Software, Engenharia de Desempenho de Software e Estudos Experimentais.

Já a área de Teste de Desempenho de Software tem intersecção com Engenharia de Desempenho e Avaliação de Desempenho, que são as mesmas intersecções da área de Engenharia de Desempenho de Software. E a área de Estudos Experimentais tem intersecção com Avaliação de Desempenho.

Também é possível observar que existem intersecções com três das áreas envolvidas: Avaliação de Desempenho, Estudos Experimentais e Teste de Desempenho de Software; e Avaliação de Desempenho, Teste de Desempenho de Software e Engenharia de Desempenho de Software. Por fim, existe uma área de intersecção que compreende todas as áreas envolvidas, que contém apenas um termo, o conceito de métricas.

Com isso é possível perceber que existe uma relação entre termos de diferentes áreas da computação, que tratam o desempenho de sistemas computacionais, já que foi mostrado que os mesmos termos são usados nessas diferentes áreas, sendo possível a unificação.

\section{Trabalhos Futuros}

As próximas etapas do projeto são relacionar os conceitos pertencentes a elas, de modo a reuni-los usando ontologias.

Dessa forma, aqueles comuns deverão ser unificados, para que um framework para se realizar uma análise de desempenho possa ser produzido, utilizando-se da convergência de conceitos realizada. Este framework de análise de desempenho contará com os métodos de experimentação, sendo adicionado nesta proposta conceitos de teste de software, mais especificamente dos critérios de teste. 


\section{Conclusões}

Existem diversas áreas de estudo da Ciência da Computação, que consideram o desempenho de sistemas computacionais, sendo elas: Avaliação de Desempenho, Teste de Desempenho de Software, Engenharia de Desempenho de Software e Estudos Experimentais. Embora tais áreas tenham objetivos diferentes, é possível perceber que elas tem pelo menos um objetivo em comum, que é a análise de desempenho de sistemas computacionais.

Porém, como são áreas diferentes, cada uma delas tratam este conceito de maneiras distintas, com diferentes termos e métodos, com um vocabulário diferente para cada uma delas. Sendo assim, surge a necessidade de unificação destes conceitos de modo a evitar replicação de esforços de especialistas dessas diferentes áreas, para que um possa compreender o outro.

A fim de mostrar que tal unificação de conceitos é possível, este artigo mostrou como tais áreas podem ser relacionadas. Para isto, foi feito um estudo da bibliografia de cada área relacionada, a partir de indicações de especialistas de cada uma delas. A partir de então foram montados mapas conceituais para identificar termos de cada uma das áreas. Então, uma lista com todos os termos que aparecem nessas mapas foi feita, e aqueles que se repetiam em mais de uma área eram apontados.

\section{Agradecimentos}

Os autores agradecem à CAPES, CNPq e FAPESP pelo apoio financeiro.

\section{Referências}

Cortellessa, V. (2005). How far are we from the definition of a common software performance ontology? In Proceedings of the 5th international workshop on Software and performance, pages 195-204. ACM.

Freitas, A. and Vieira, R. (2014). An ontology for guiding performance testing. In Proceedings of the 2014 IEEE/WIC/ACM International Joint Conferences on Web Intelligence (WI) and Intelligent Agent Technologies (IAT)-Volume 01, pages 400-407. IEEE Computer Society.

Jain, R. (1991). The art of computer system performance analysis: techniques for experimental design, measurement, simulation and modeling. New York: John Willey.

Sancho, P. P., Juiz, C., Puigjaner, R., Chung, L., and Subramanian, N. (2007). An approach to ontology-aided performance engineering through nfr framework. In Proceedings of the 6th international workshop on Software and performance, pages 125-128. ACM.

Smith, C. U. (1986). The evolution of software performance engineering: a survey. In Proceedings of 1986 ACM Fall joint computer conference, pages 778-783. IEEE Computer Society Press.

Wohlin, C., Runeson, P., Höst, M., Ohlsson, M. C., Regnell, B., and Wesslén, A. (2012). Experimentation in software engineering. Springer.

Woodside, M., Franks, G., and Petriu, D. C. (2007). The future of software performance engineering. In Future of Software Engineering, 2007. FOSE'07, pages 171-187. IEEE. 